Article

\title{
Nonlinear dynamics of MEMS resonator in PLL-AGC self-oscillation loop
}

\author{
Aleksei Lukin $1, * \mathbb{C}$, Yakov Belyaev ${ }^{2,+}$, Dmitry Indeitsev ${ }^{1,3,+}$ and Ivan Popov ${ }^{1, \dagger}$ \\ 1 Peter the Great St.Petersburg Polytechnic University, St.Petersburg, Russia; lukinalexei@yandex.ru \\ 2 Concern CSRI Elektropribor, St.Petersburg, Russia; ybelyaev@eprib.ru \\ 3 Institute for Problems in Mechanical Engineering of the Russian Academy of Sciences, St.Petersburg, Russia; \\ dmitry.indeitsev@gmail.com \\ * Correspondence: lukinalexei@yandex.ru \\ + These authors contributed equally to this work.
}

\begin{abstract}
The work is devoted to the study of a MEMS resonator dynamics under the action of phase-locked and automatic gain control loops. Particular attention is directed to the study of the nonlinearity factor of the resonator elastic restoring force. It was found that the determination of control system parameters based on the stability analysis of the operating resonant mode, in the general case, does not provide the required phase adjustment and stabilization of the oscillation amplitude. Stable multifrequency modes of oscillations are found, an analytical study of the mechanisms of their appearance and evolution is carried out under variation of the key parameters of the system. The real regions of the control system stable operation are determined (which do not coincide, as was found, with the regions of stability of the operating resonant mode, due to the presence of hidden attractors in the phase space of the system). A methodology has been developed for identifying such areas of stable operation. A significant complication of the structure of possible motions in the system with an increase in the Q-factor of the resonator is revealed.
\end{abstract}

Keywords: MEMS resonator; nonlinear dynamics; phase-locked loop (PLL); automatic gain control (AGC); hidden attractors

\section{Introduction}

High requirements for the stability of the operation of resonant sensors (in particular, the sensitive elements of microelectromechanical vibration gyroscopes) lead to the need to use feedback control systems that ensure the constancy of the oscillation amplitude of the resonator and its frequency tuning to resonance. The first of these tasks, as a rule, is solved using the automatic gain control (AGC) system [1-4]. To solve the second problem, phase-locked loop (PLL) systems [5-7] are widely used.

Mathematical modeling of the dynamics of MEMS resonators considering operation of closed-loop control systems is of significant theoretical and applied interest. A large number of scientific works are devoted to various aspects of this problem. In [8], an analytical study of stationary oscillatory regimes for a mechanically linear resonator was carried out taking into account the action of the AGC system. In the same work, the dynamics of a system of two weakly coupled oscillators in a control loop is considered. In [9] the dynamics of the resonator under the action of the PLL is studied. The region of stability of the required resonant oscillation mode in the parameter space of the control system is found. Calculations of the resonator dynamics are performed by a direct numerical method, taking into account the nonlinearity of the elastic restoring force. A qualitative study of the dynamics of a mechanically linear resonator with the combined action of the AGC and PLL systems was carried out in [10]. This work also considers the issues of tuning the natural frequency of the resonator, the generation of primary oscillations for a two-mass vibration gyroscope and the adjustment of the frequencies of its primary and secondary oscillations. The mathematical apparatus of these works are applied methods of nonlinear oscillations theory (in most cases, the averaging method). 
A separate area of research is the study of essentially nonlinear effects characteristic to the considered control algorithms and controlled systems in a whole. In a number of works, the nonlinear dynamics and bifurcations of the operating modes of the PLL circuit [11,12] are studied. The existence of hidden attractors in the dynamics of the PLL is revealed. The difficulties associated with this in the numerical simulation of the processes of interest are noted and overcome [13]. There are also known works devoted to the study of the nonlinear dynamics of MEMS resonators in control loops that implement the amplitude and phase stabilization of oscillations [14-16].

This work is devoted to a qualitative (parametric) study of the nonlinear dynamics of a MEMS resonator with a nonlinear restoring force under the combined action of the AGC and PLL systems. The issues of stability of stationary oscillatory modes, their evolution and branching are considered depending on the key mechanical parameters of the resonator and parameters of the control system.

\section{Mathematical model}

Let us investigate the dynamics of a micromechanical resonator under the action of a control system designed for resonant frequency tuning and stabilization of the vibration amplitude. Tuning the frequency of the electrostatic comb-drive to resonance is provided by a phase-locked loop system. Stabilization of the amplitude of the primary oscillations is achieved by the operation of the automatic gain control loop.

The PLL circuit contains a phase detector, a low-pass filter (LPF), a controller and a voltage-controlled oscillator (VCO) [7,17]. The circuit is a closed-loop control system whose task is to provide the required phase difference between the output signal of the capacitance-voltage converter of the resonator displacement sensor and the VCO reference signal.

The amplitude channel, which implements the AGC circuit, consists of an amplitude detector and a PI controller.

The mathematical model of the resonator in the PLL-AGC system is described by the following system of equations $[9,10]$ :

$$
\begin{array}{r}
\ddot{x}+c \dot{x}+\omega_{n}^{2} x+\beta x^{3}=A \cos \theta \\
\dot{\theta}=\omega_{0}+K_{V C O} z \\
\dot{z}=K_{I}^{P L L} y \\
\dot{y}=\lambda^{P L L}\left(K_{G} x \cos \theta-y\right) \\
A=K_{p}\left(X_{0}-r\right)+B \\
\dot{B}=K_{I}^{A G C}\left(X_{0}-r\right) \\
\dot{r}=\lambda^{A G C}\left(\frac{\pi}{2}|x|-r\right),
\end{array}
$$

where $x$-displacement of the resonator, $\omega_{n}$ - natural frequency of the resonator, $c=2 \omega_{n} \xi=\omega_{n} / Q$ - dissipation parameter ( $\xi$ - coefficient of relative attenuation, $Q$ - quality factor of the resonator), $\beta$ - coefficient for the cubic term in the restoring force, $\theta$ - phase of the VCO $(\omega=\dot{\theta}$ - instantaneous VCO frequency), $z$ - VCO control signal, $y$ - output signal of the phase detector, $\omega_{0}-$ natural (free) frequency of the VCO, $K_{V C O}$ - proportional gain of the VCO, $K_{I}^{P L L}$ - integral gain of the controller, $K_{G}, \lambda^{P L L}-$ transmission coefficient and frequency of the PLL low-pass filter, $A$ - output signal of the AGC circuit, $B$ - control signal of the AGC, $r$ - estimate of the current oscillation amplitude, $K_{p}, K_{I}^{A G C}$ - proportional and integral coefficients of the controller of the AGC circuit, $\lambda^{A G C}-$ frequency of the pole of the AGC low-pass filter, $X_{0}$ - required amplitude of stationary oscillations.

It is convenient to associate the parameter of the geometric nonlinearity of the resonator $\beta$ with the ratio $\kappa$ of the linear and cubic components of the elastic restoring force at the displacement amplitude $X_{0}$ : 


$$
\begin{array}{r}
\beta X_{0}^{3}=\kappa \omega_{n}^{2} X_{0}, \\
\beta=\kappa \frac{\omega_{n}^{2}}{X_{0}^{2}} .
\end{array}
$$

\section{Qualitative analysis of the system nonlinear dynamics}

For a qualitative (parametric) study of the dynamics of the system, we will apply the averaging method. We represent the sought solution for the mechanical degree of freedom in the form

$$
x=a(t) \cos [\theta(t)+\phi(t)],
$$

where $a(t)$ - amplitude, $\theta(t)+\phi(t)$ - instantaneous phase of oscillations. Instantaneous VCO frequency is $\theta$, thus $\phi$ is phase difference between the resonator and the VCO.

Differentiating (3), we get

$$
\dot{x}=-a \dot{\theta} \sin (\theta+\phi)+\dot{a} \cos (\theta+\phi)-a \dot{\phi} \sin (\theta+\phi) .
$$

Assume that

$$
\dot{a} \cos (\theta+\phi)-a \dot{\phi} \sin (\theta+\phi)=0
$$

then

$$
\ddot{x}=-\dot{a} \dot{\theta} \sin (\theta+\phi)-a \ddot{\theta} \sin (\theta+\phi)-a \dot{\theta}(\dot{\theta}+\dot{\phi}) \cos (\theta+\phi) .
$$

Note that the VCO phase dynamics is described by simple equation:

$$
\ddot{\theta}=K_{V C O} K_{I}^{P L L} y \text {. }
$$

The variable $\theta$ is "fast" and can be thought of as normalized time. Following the procedure of the averaging method, substitute (3), (4) and (6) into (1), express from the first equation (1) and (5) $\dot{a}, \dot{\phi}$ and average the right parts of the system over the explicitly incoming "time" $\theta$. Thus, we come to an autonomous system of differential equations describing the evolution of slow variables $\tilde{a}, \tilde{\phi}, \tilde{z}, \tilde{y}, \tilde{B}, \tilde{r}$ :

$$
\begin{array}{r}
\dot{\tilde{a}}=-\frac{\tilde{B} \sin \tilde{\phi}+K_{p}\left(X_{0}-\tilde{r}\right) \sin \tilde{\phi}+c\left(\omega_{0}+K_{V C O} \tilde{z}\right) \tilde{a}+K_{V C O} K_{I}^{P L L} \tilde{y} \tilde{a}}{2\left(\omega_{0}+K_{V C O} \tilde{z}\right)} \\
\dot{\tilde{\phi}}=-\frac{\left[\left(\omega_{0}+K_{V C O} \tilde{z}\right)^{2}-\omega_{n}^{2}\right] \tilde{a}+\left[\tilde{B}+K_{p}\left(X_{0}-\tilde{r}\right)\right] \cos \tilde{\phi}-\frac{3}{4} \beta \tilde{a}^{3}}{2 \tilde{a}\left(\omega_{0}+K_{V C O} \tilde{z}\right)} \\
\dot{\tilde{y}}=-\lambda^{P L L}\left(\tilde{y}-\frac{K_{G}}{2} \tilde{a} \cos \tilde{\phi}\right) \\
\dot{\tilde{B}}=K_{I}^{P L C} \tilde{y} \\
\dot{\tilde{r}}=\lambda^{A G C}\left(X_{0}-\tilde{r}\right)
\end{array}
$$

Possible stationary periodic regimes of the system (1) correspond to fixed points (equilibrium positions) of the dynamical system (8):

$$
\tilde{y}_{0}=0, \quad \tilde{r}_{0}=X_{0}, \quad \tilde{a}_{0}=X_{0}, \quad \tilde{\phi}_{0}= \pm \frac{\pi}{2} .
$$

The stationary values $\tilde{z}$ are determined from the equation 


$$
\left(\omega_{0}+K_{V C O} \tilde{z}_{0}\right)^{2}-\omega_{n}^{2}-\frac{3}{4} \beta X_{0}^{2}=0
$$

Finally,

$$
\tilde{B}_{0}=c X_{0}\left[\omega_{0}+K_{V C O} \tilde{z}_{0}\right]
$$

The main interest is a solution for which $\tilde{\phi}_{0}=-\frac{\pi}{2}$ and $\tilde{z}_{0}$ has the sign of $\omega_{n}-\omega_{0}$.

\subsection{Stability analysis of stationary regimes}

The stability analysis of the considered stationary regime is performed by calculating the eigenvalues of the Jacobian of the right-hand side of the system (8) at a given fixed point. For a linear resonator $(\kappa=0)$, the area of stability in the parameter space is characterized by the following conditions [10]:

$$
\begin{array}{r}
K_{I}^{A G C}<K_{I, M A X}^{A G C}=\omega_{n}\left(c+\frac{K_{p}}{\omega_{n}}\right) \cdot\left(\frac{c}{2}+\lambda^{A G C}\right) \\
K_{I}^{P L L}<K_{I, M A X}^{P L L}=\frac{c\left(\lambda^{P L L}+\frac{c}{2}\right)}{K_{G} K_{V C O} X_{0}} .
\end{array}
$$

For the general case $\kappa \neq 0$, the derived conditions characterizing the stability of the system are quite cumbersome and are not explicitly given here.

Among the parameters of the system (8), let us single out the groups of fixed and variable. The values of the fixed parameters are given in Table 1.

Table 1. Values of fixed system parameters

\begin{tabular}{cc}
\hline Parameter & Value \\
\hline$f_{n}\left(\omega_{n}=2 \pi f_{n}\right)$ & $10 \mathrm{kHz}$ \\
$f_{0}\left(\omega_{0}=2 \pi f_{0}\right)$ & $9 \mathrm{kHz}$ \\
$X_{0}$ & $3 \mu \mathrm{m}$ \\
$K_{p}$ & $10^{8}$ \\
$K_{G}$ & $10^{6}$ \\
$K_{V C O}$ & $10^{4}$ \\
\hline
\end{tabular}

Variable parameters are $Q, \kappa, \lambda^{A G C}, \lambda^{P L L}, K_{I}^{A G C}, K_{I}^{P L L}$.

\subsubsection{Case of mechanically linear resonator}

Let us present the results of numerical integration of the initial (1) and averaged (8) systems describing the dynamics of the resonator for the parameter values $K_{I}^{A G C}, K_{I}^{P L L}$ equal to 0.9 and 1.1 of the corresponding critical values calculated from (12). It is assumed that $Q=100, \kappa=0, \lambda^{A G C}=$ $\lambda^{P L L}=f_{n} / 10$. The quantities characterizing the dynamics of the resonator and the operation of the AGC and PLL circuits are shown in Figures 1, 2a and 2b, respectively. Red color denotes the solution of the averaged system in slow variables, blue - the solution of the original system. 


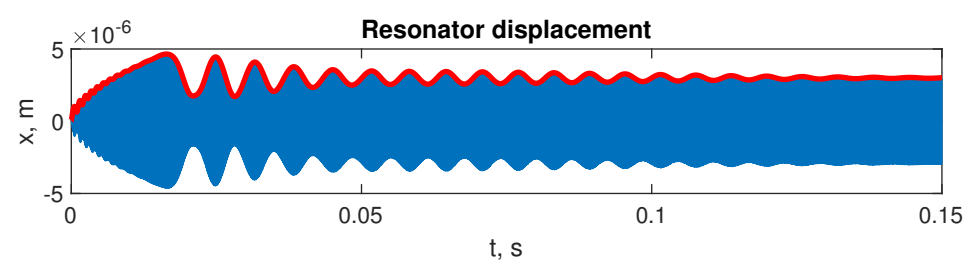

Relative phase angle
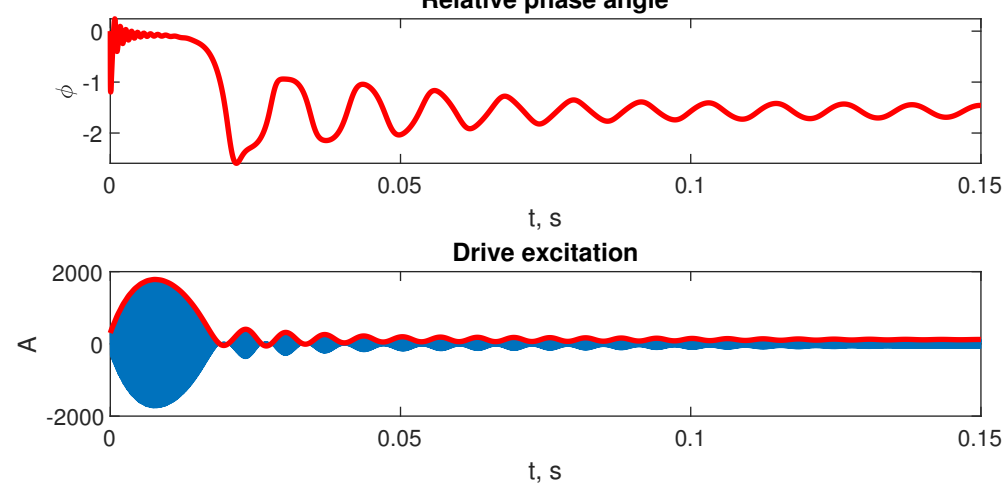

Figure 1. Dynamics of the resonator. $K_{I}^{A G C}=0.9 K_{I, M A X^{\prime}}^{A G C} K_{I}^{P L L}=0.9 K_{I, M A X}^{P L L}$
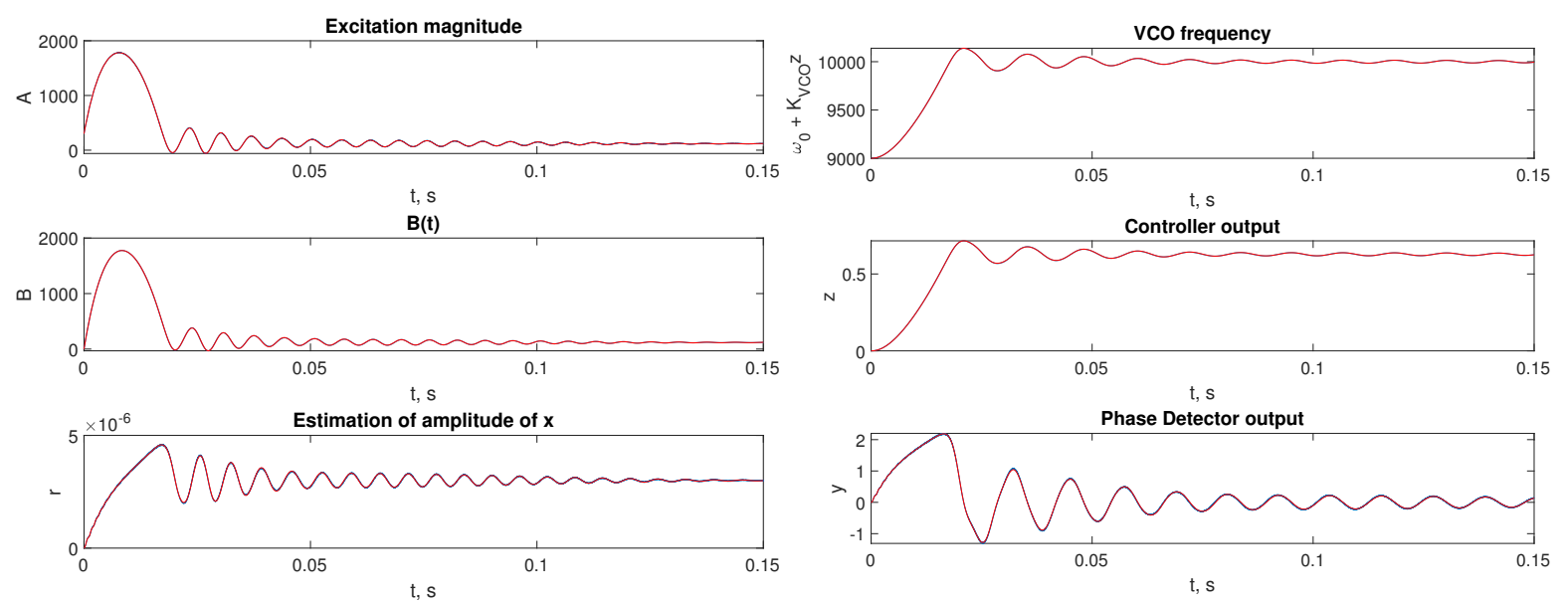

(a) AGC loop

(b) PLL loop

Figure 2. Control circuit dynamics. $K_{I}^{A G C}=0.9 K_{I, M A X}^{A G C}, K_{I}^{P L L}=0.9 K_{I, M A X}^{P L L}$

As can be seen from the figures, when the stability conditions of the control system are satisfied, the resonator reaches the resonant oscillation mode with the required amplitude. At the same time, equations in slow variables allow one to simulate the dynamics of a system accurately and much more efficiently from a computational point of view, in comparison with direct integration.

The following Figures 3, 4 show the calculation results for the values of the control system parameters corresponding to the instability region of the AGC loop. 


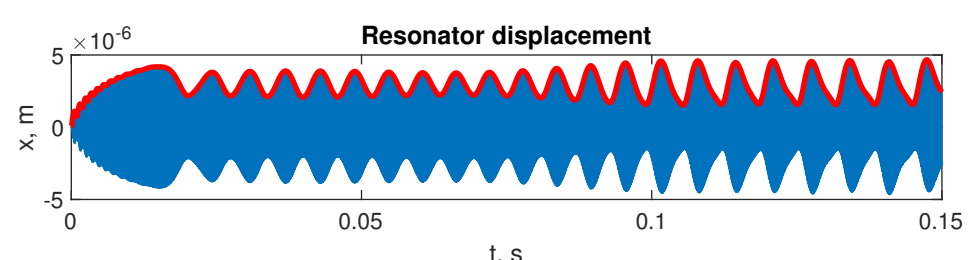

t, $s$
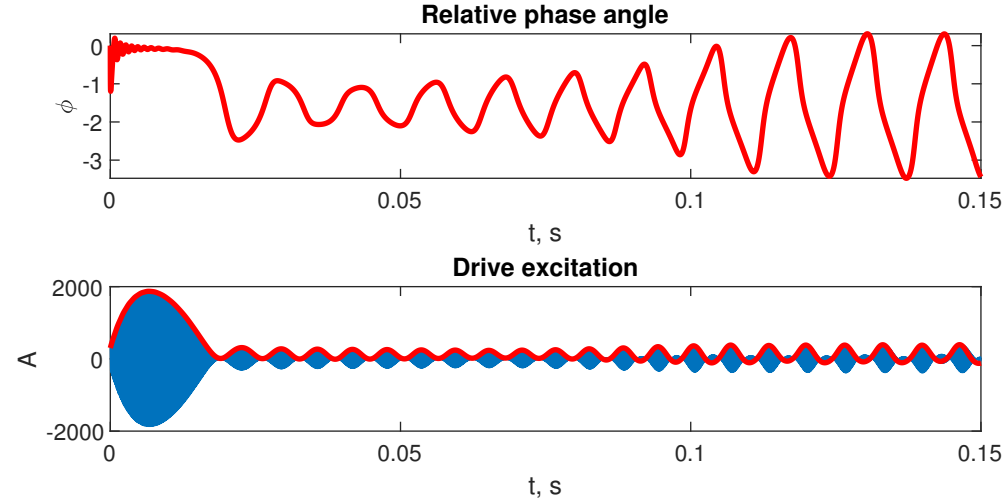

Figure 3. Dynamics of the resonator. $K_{I}^{A G C}=1.1 K_{I, M A X^{\prime}}^{A G C} K_{I}^{P L L}=0.9 K_{I, M A X}^{P L L}$
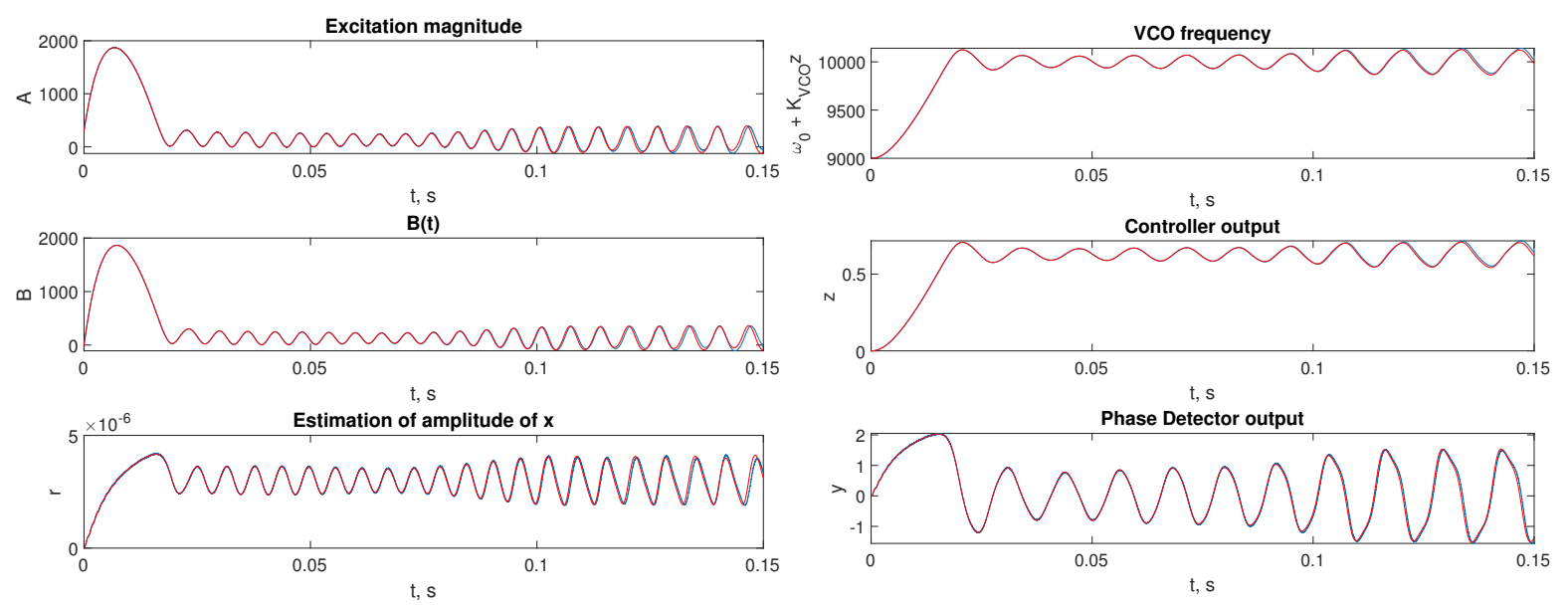

(a) AGC loop

(b) PLL loop

Figure 4. Control circuit dynamics. $K_{I}^{A G C}=1.1 K_{I, M A X}^{A G C}, K_{I}^{P L L}=0.9 K_{I, M A X}^{P L L}$

As can be seen from the figures, going out of the stability region in terms of the parameters of the AGC circuit leads to oscillations of the resonator with variable amplitude, i.e. to incorrect operation of the control system.

Similar results for the case of going beyond the boundary of the stability region in terms of the parameters of the PLL circuit are shown in Figures 5, 6. 

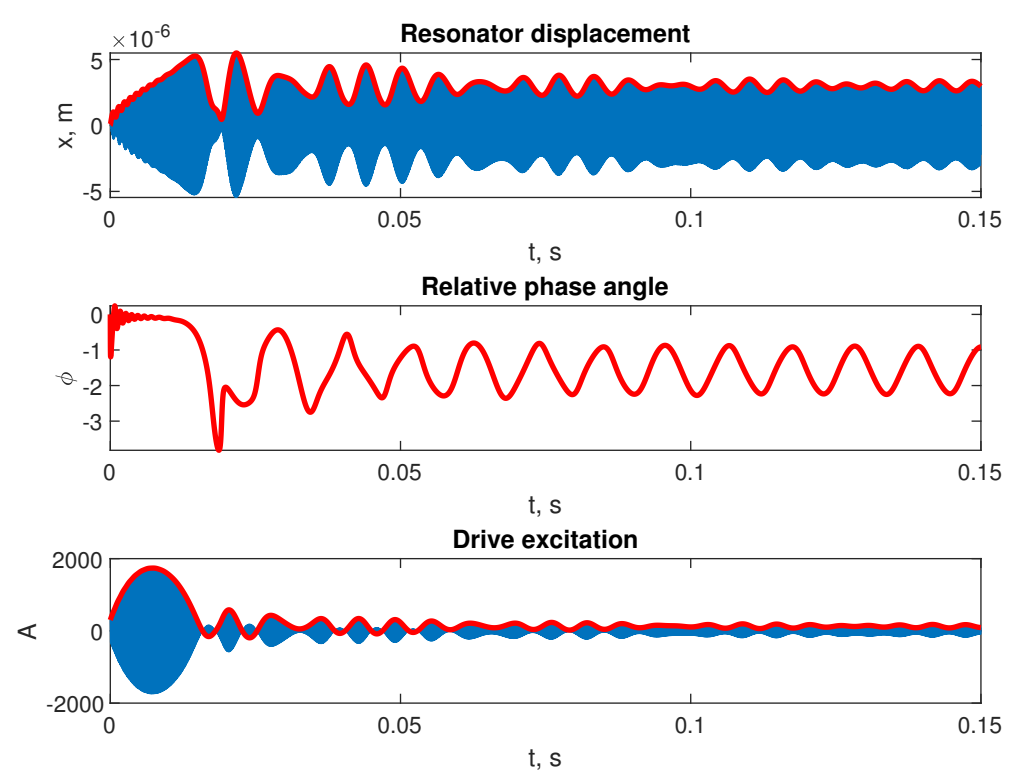

Figure 5. Dynamics of the resonator. $K_{I}^{A G C}=0.9 K_{I, M A X}^{A G C}{ }^{\prime} K_{I}^{P L L}=1.1 K_{I, M A X}^{P L L}$
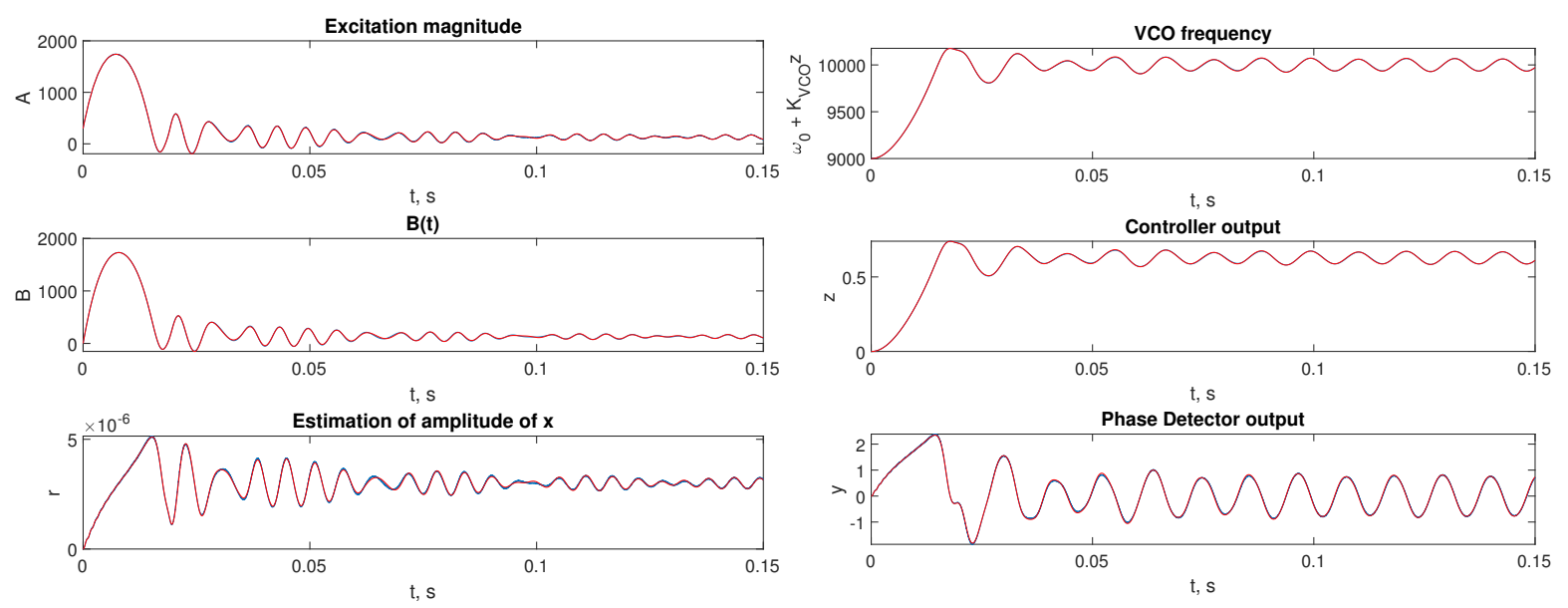

(a) AGC loop

(b) PLL loop

Figure 6. Control circuit dynamics. $K_{I}^{A G C}=0.9 K_{I, M A X}^{A G C}, K_{I}^{P L L}=1.1 K_{I, M A X}^{P L L}$

Leaving the stability region in terms of the parameters of the PLL circuit also leads to oscillations of the resonator with variable amplitude. As in the previous case, at the end of the transient process, the system enters a two-frequency stationary oscillation mode in the vicinity of the required resonant mode.

Everywhere below, we take $K_{I}^{A G C}=0.9 K_{I, M A X}^{A G C}, K_{I}^{P L L}=0.9 K_{I, M A X}^{P L L}$. Also, unless otherwise stated, we assume $\lambda^{A G C}=\lambda^{P L L}=f_{n} / 10$.

\subsubsection{Taking into account the nonlinearity of the restoring force}

Further stages of the study will be devoted to the analysis of the influence of the nonlinearity factor of the resonator $\kappa$ and the peculiarities of the system dynamics for various values of the quality factor $Q$.

Let us investigate the stability of the required resonant operation mode with respect to changes in the parameter $\kappa$. Figure 7 shows the dependence of the maximum real part among all eigenvalues for the investigated fixed point of the system (8) depending on $\kappa$ for $Q=10,100,1000$. 


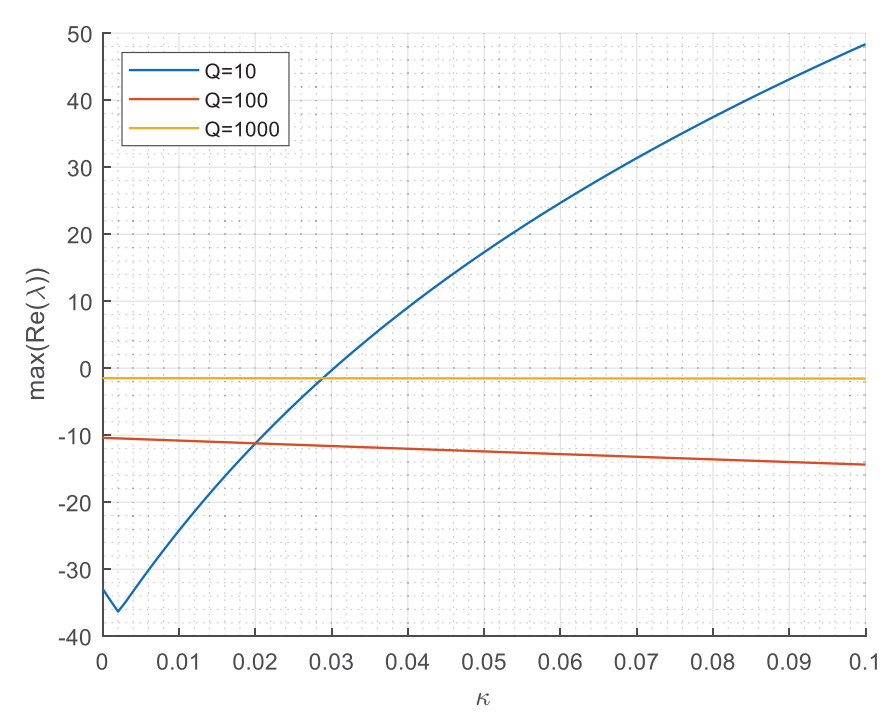

Figure 7. On the analysis of the stability of the resonant operation mode

As can be seen from the figure, for $Q=10$ at $\kappa \approx 3 \%$ there is a loss of stability of the stationary solution. For larger values of the quality factor in the considered range of $\kappa$, the stability of the control system tuned according to the linear model is not violated.

The following are simulation results for $Q=10, \kappa=2.5 \%$ (Figure 8 ) and 3.5\% (Figure 9). Two variants of initial conditions are considered - those close to the equilibrium point $\left\{\tilde{a}_{0}, \tilde{\phi}_{0}, \tilde{z}_{0}, \tilde{y}_{0}, \tilde{B}_{0}, \tilde{r}_{0}\right\}$ (Figures 8a, 9a) and close to zero in all phase variables (Figures 8b, 9b).
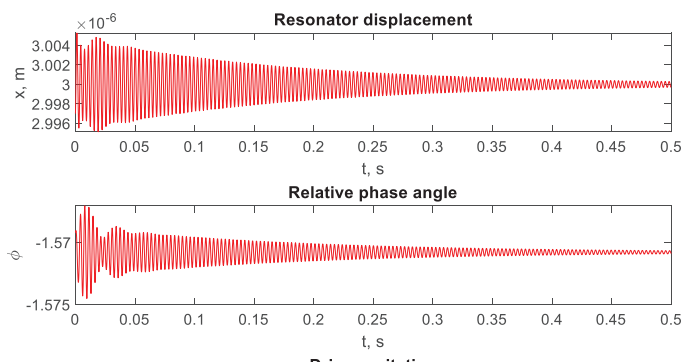

Drive excitation

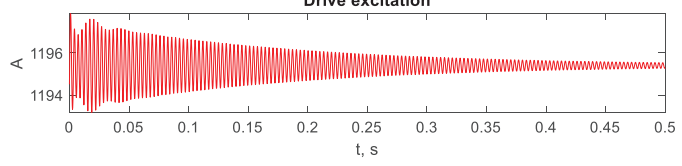

(a) I.C. close to stationary solution
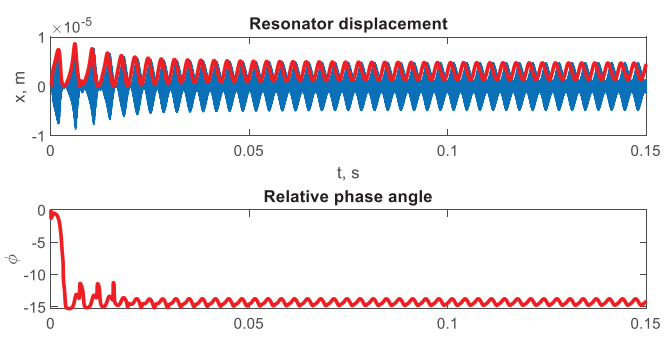

Drive

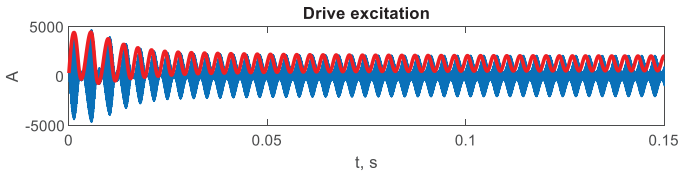

(b) Zero I.C.

Figure 8. Dynamics of the resonator. $\kappa=0.025$

As can be seen from the Figure $8 \mathrm{a}$, when choosing the initial conditions inside a small neighborhood of the stationary solution, the system actually reaches the required resonant oscillation mode. According to the Figure 8b, in spite of the fact that the steady state mode is stable for the selected parameters values, under arbitrary (in this case, zero) initial conditions, a continuous two-frequency oscillatory process is observed without reaching the required resonance mode. Hence, it can be concluded that there exist complex stable multifrequency periodic oscillation modes outside the basin of attraction of the investigated single-frequency resonant mode - hidden attractors [11,13]. 

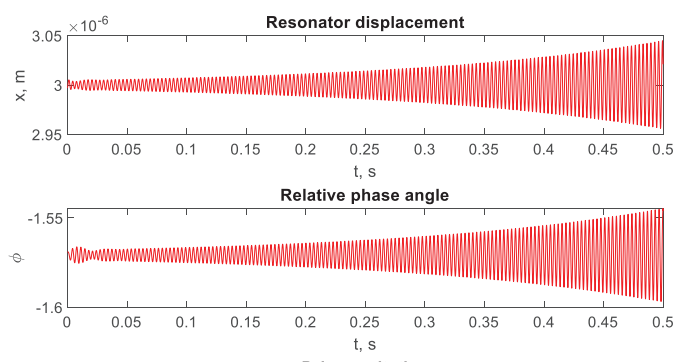
Drive excitation

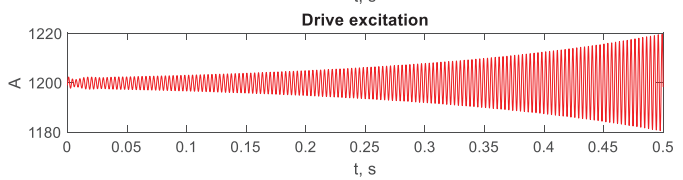

(a) I.C. close to stationary solution

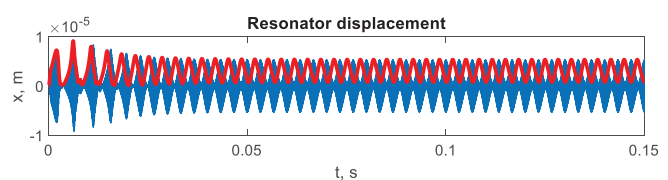

Relative phase angle
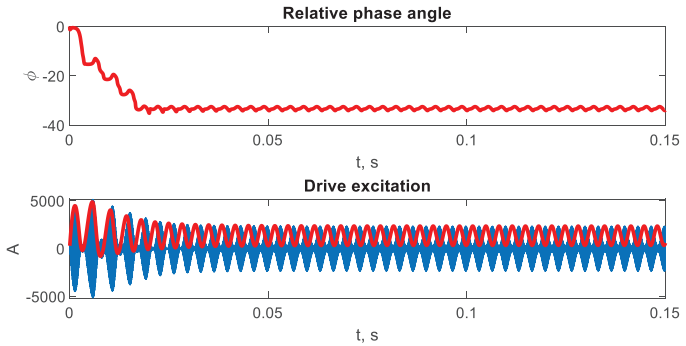

(b) Zero I.C.

Figure 9. Dynamics of the resonator. $\kappa=0.035$

As can be seen from the Figure 9a, the dynamics of the system outside the stability region of the stationary regime has the expected character of exponential growth from the initial state. According to the Figure $9 b$, under zero initial conditions for the considered values of the nonlinearity parameter, there are no qualitative differences between the behavior of the system inside and outside the stability region of the required resonant mode - in both cases we have two-frequency oscillatory mode. Thus, it can be concluded that the adjustment of the parameters of the PLL-AGC system for a nonlinear resonator based on the stability conditions for the statonary mode is not justified and, in the general case, does not lead to the required stabilization of the amplitude and phase of the resonator oscillations.

Let us now investigate the dynamics of higher-Q systems. Based on the Figure 7, we see that for $Q=100$ in the range of values $\kappa$ to $10 \%$, the stability of the stationary mode is preserved. Hereinafter, the calculations will be performed for zero initial conditions (i.e., outside, as will be shown below, a very narrow basin of attraction of single-frequency motion). Figures 10, 11 and 12, 13 show the simulation results for the values $\kappa$ equal to 2 and $2.5 \%$ respectively.
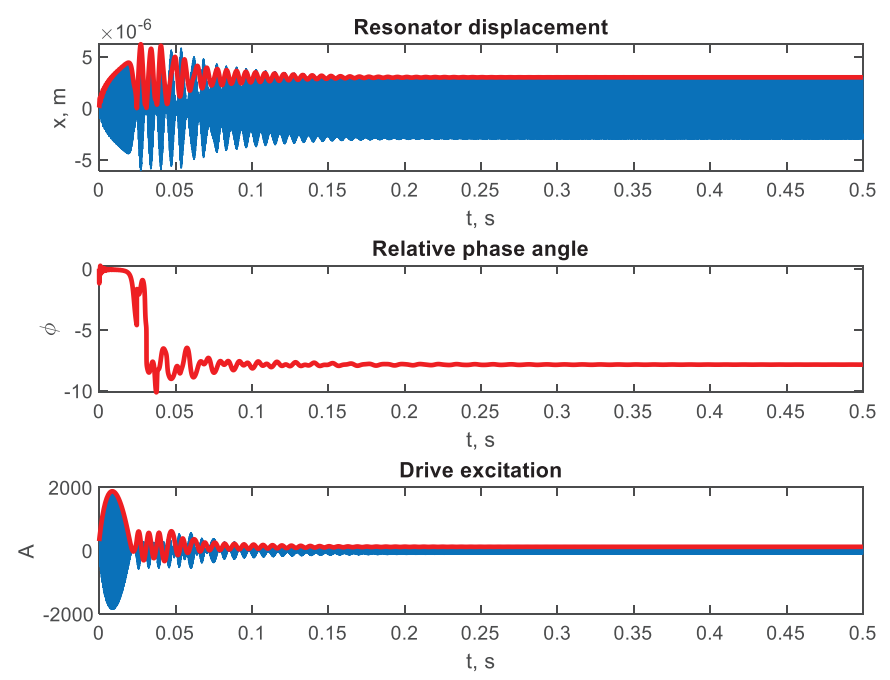

Figure 10. Dynamics of the resonator. $\kappa=2 \%$ 


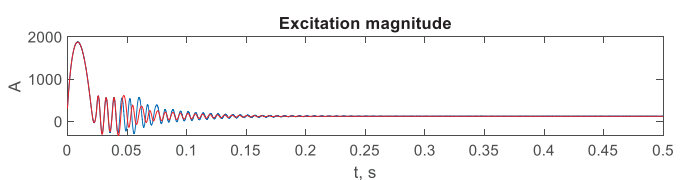

$B(t)$
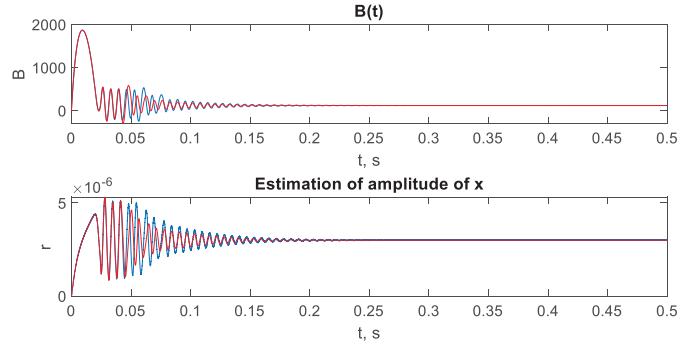

(a) AGC loop
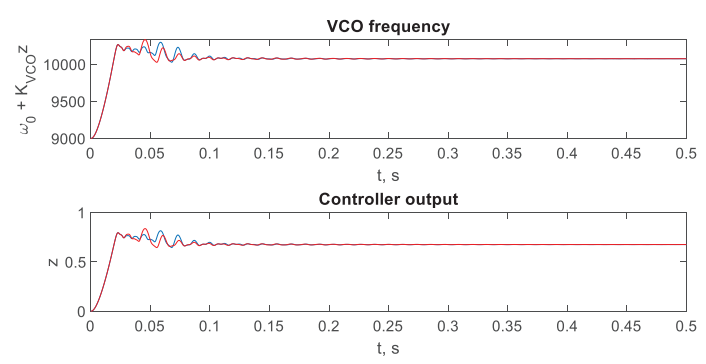

Phase Detector output

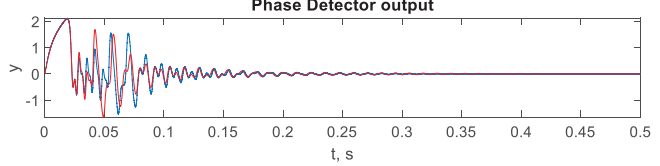

(b) PLL loop

Figure 11. Control circuit dynamics. $\kappa=2 \%$

As can be seen from the figures, at $\kappa=2 \%$ the control system provides stabilization of the oscillation amplitude of the resonator and its resonance tuning.
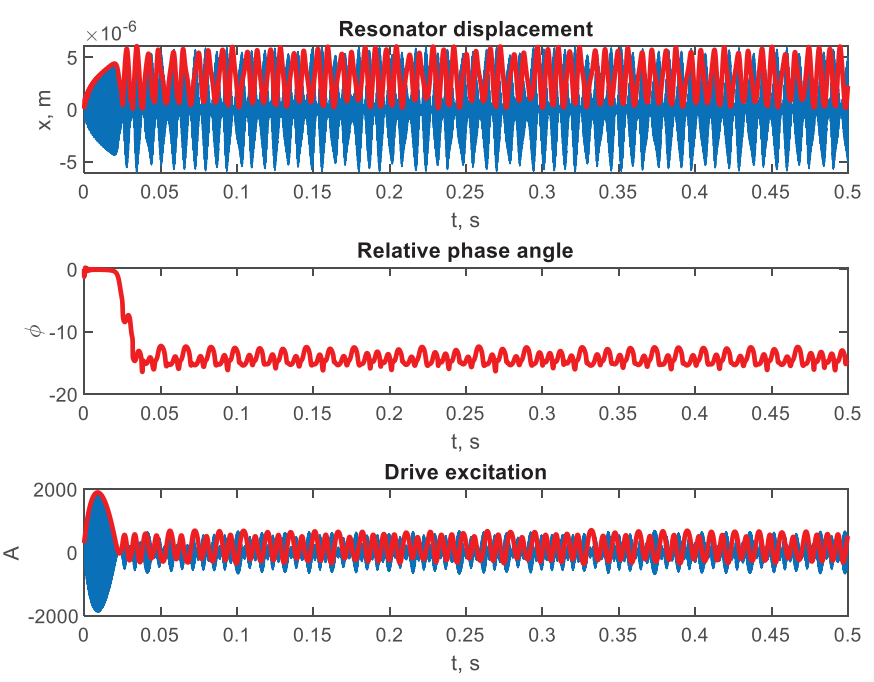

Figure 12. Dynamics of the resonator. $\kappa=2.5 \%$
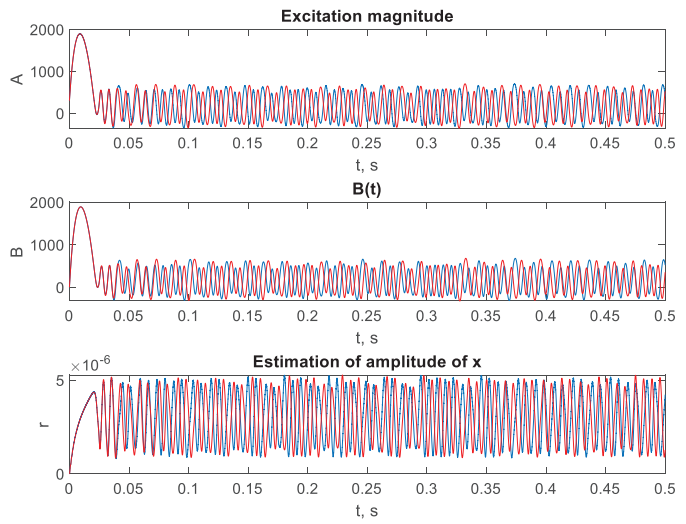

(a) AGC loop

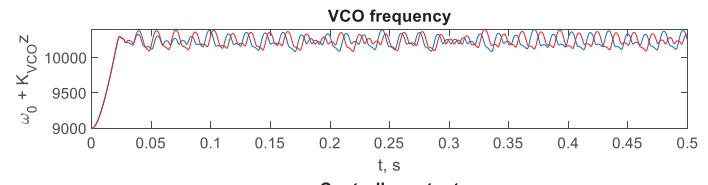

Controller output
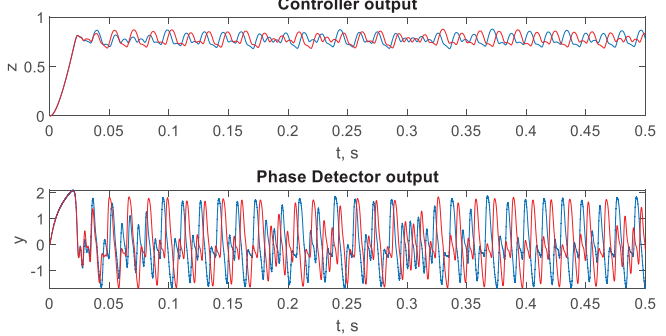

(b) PLL loop

Figure 13. Control circuit dynamics. $\kappa=2.5 \%$ 
From the figures above it can be seen that at $\kappa=2.5 \%$ the system enters a complex multifrequency mode of motion despite the predictions of stability analysis. Physically, the solution obtained corresponds to the hysteresis motion of the representing point in the "amplitude-frequency" space according to the resonance (amplitude-frequency) characteristic of the mechanical resonator - due to nonlinearity, the skeletal curve has a slope to the right, which leads to the possibility of the phenomenon of "breakdowns" of oscillations when passing through the corresponding limit points.

\subsection{Evolution and branching of steady-state oscillatory regimes}

From a practical point of view, it is important to determine the areas in the parameter space of the mechanical structure and control system, corresponding to the possibility of maintaining the required resonant oscillation mode for a sufficiently wide range of initial conditions and amplitudes of external disturbing influences (temperature instability, vibrations, shocks and acoustic noise).

Such an analysis can be carried out using applied methods of nonlinear dynamics and the theory of bifurcations. Let us consider the specific task of determining the minimum value of the nonlinearity parameter $\kappa$, for which the described above undesirable oscillatory modes are possible. The calculations will be carried out for different values of the quality factor $Q$. We assume other parameters of the system to be fixed.

\subsubsection{Factor of mechanical nonlinearity}

As can be seen from Figure $8 \mathrm{~b}$, for $Q=10$ the two-frequency oscillatory mode is observed at $\kappa=2.5 \%$. This solution is a stable (attractive) limit cycle. For such objects of the phase space of a dynamical system, numerical-analytical methods have been developed that allow the continuation of the solution by the parameters of interest. The numerical implementation of these algorithms is performed in the MATCONT [18] software package. Let us graphically represent the evolution of the limit cycle when $\kappa$ changes in the subspace $\{\tilde{a}, \tilde{\phi}\}$ (Figure 14).

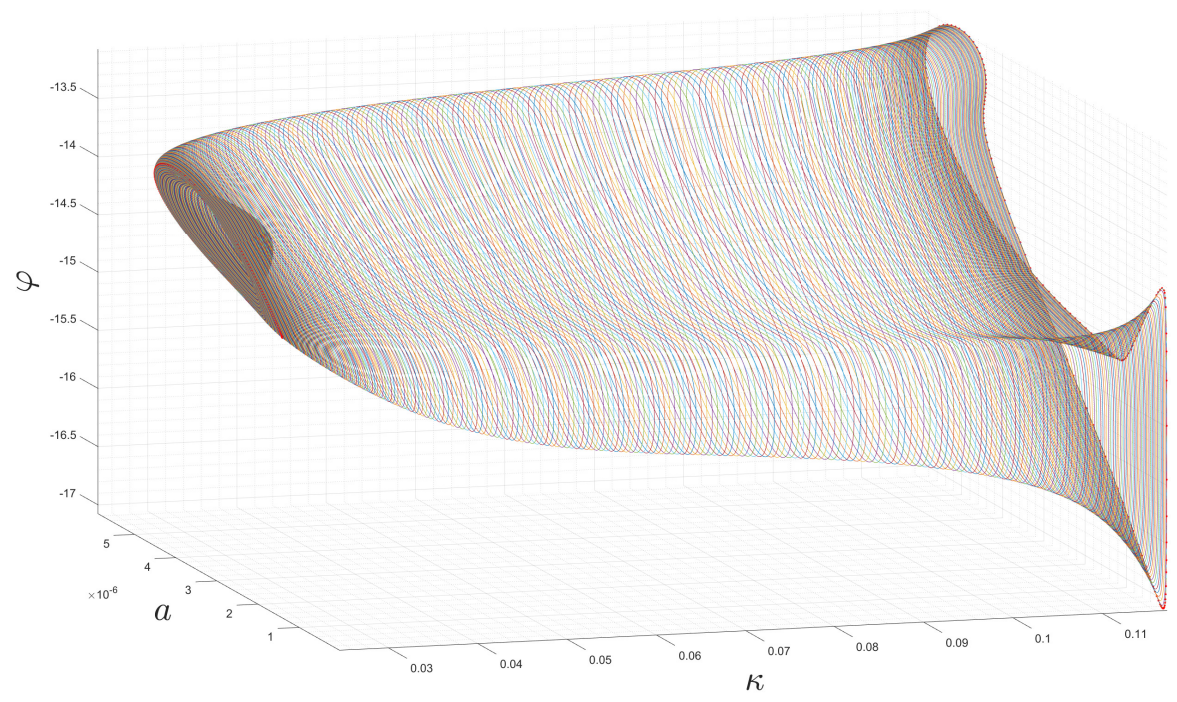

Figure 14. Evolution of the limit cycle while changing $\kappa$

We see that at $\kappa \approx 2.5 \%$ the fold bifurcation of the limit cycle occurs - at values $\kappa<2.5 \%$ the undesirable oscillatory mode does not exist; as $\kappa$ grows, the nature of the two-frequency mode becomes more complicated, the amplitude of oscillations around the required value $\left\{\tilde{a}_{0}, \tilde{\phi}_{0}\right\}$ increases. This is illustrated in Figures 15a-15b, where the deviations of the amplitude and phase of the oscillations from the corresponding resonance (required) values are shown. 


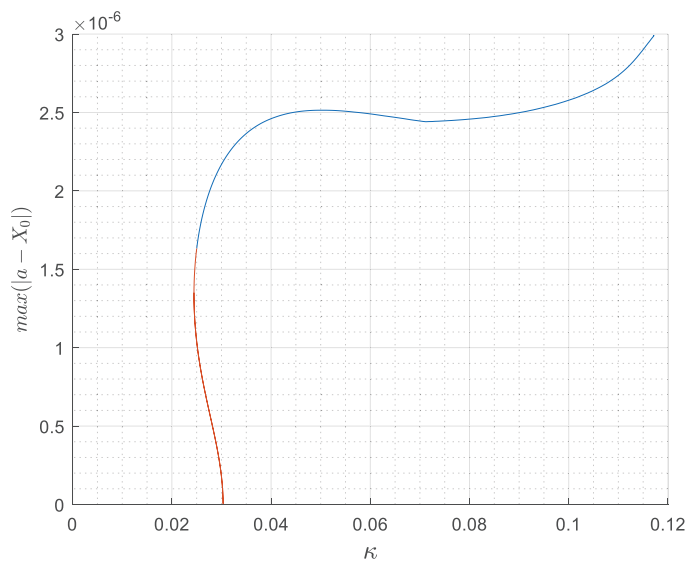

(a) Amplitude deviation

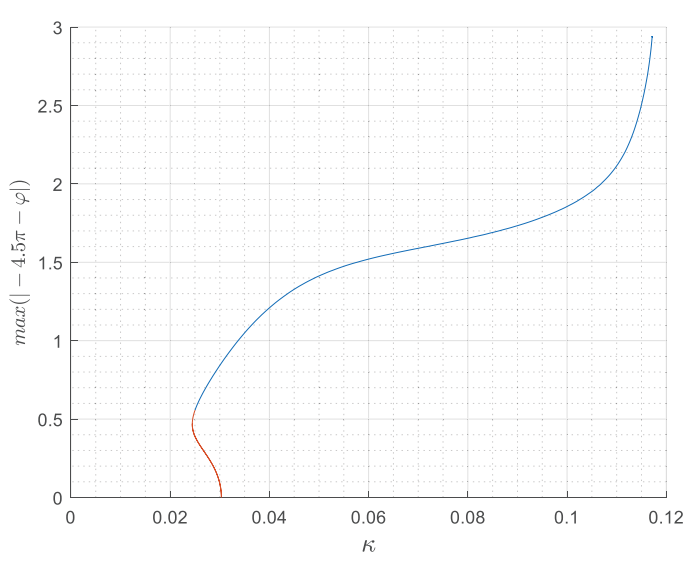

(b) Phase deviation

Figure 15. Deviations of observed dynamics from the required resonant operation mode

Note that in accordance with the Figure 7, for $\kappa=3 \%$ the Andronov-Hopf bifurcation is observed in the system - from a stable stationary resonant regime with an amplitude $\tilde{a}_{0}$ an unstable limit cycle branches off into the region of smaller $\kappa$, becoming stable through the aforementioned fold bifurcation; the stationary regime itself at $\kappa>3 \%$ is unstable. These features of the system explain the numerically observed fact presented above that undesirable oscillatory modes can also arise when the condition for the stability of the required resonant mode is met - the reason is the narrowness of the basin of attraction of this solution and the presence of an adjacent stable multifrequency motion.

A similar calculation was carried out for $Q=30$. The figure 16 shows the evolution of the limit cycle when $\kappa$ changes in the subspace $\{\tilde{a}, \tilde{\phi}\}$.

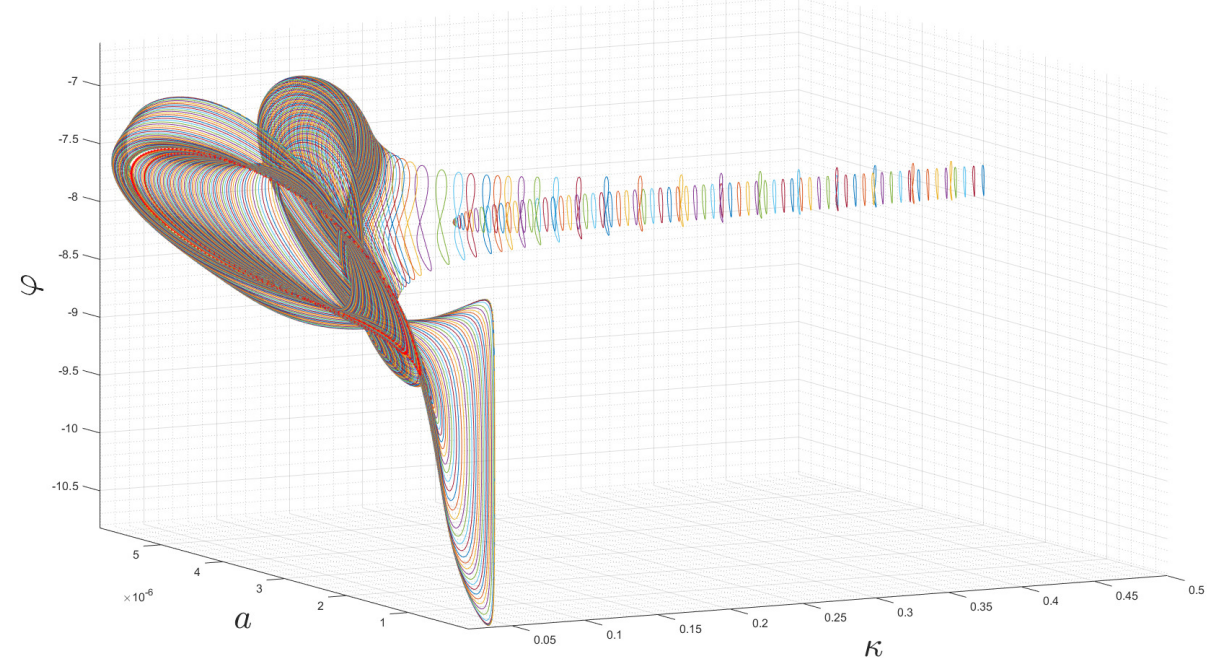

Figure 16. Evolution of the limit cycle while changing $\kappa$

As can be seen from Figure 16, with an increase in the Q-factor of the resonator, the structure of undesirable dynamic modes in the system becomes much more complicated; there is a multiplicity of possible multifrequency modes with one value of the nonlinearity parameter; their amplitude is especially large in the region of small $\kappa$.

Figures $17 \mathrm{a}-17 \mathrm{~b}$ show the deviations of the amplitude and phase of the oscillations from the required resonance values. 


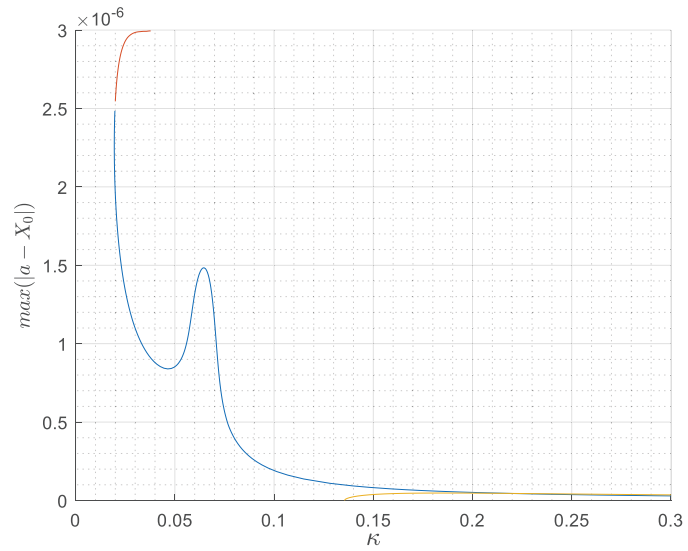

(a) Amplitude deviation

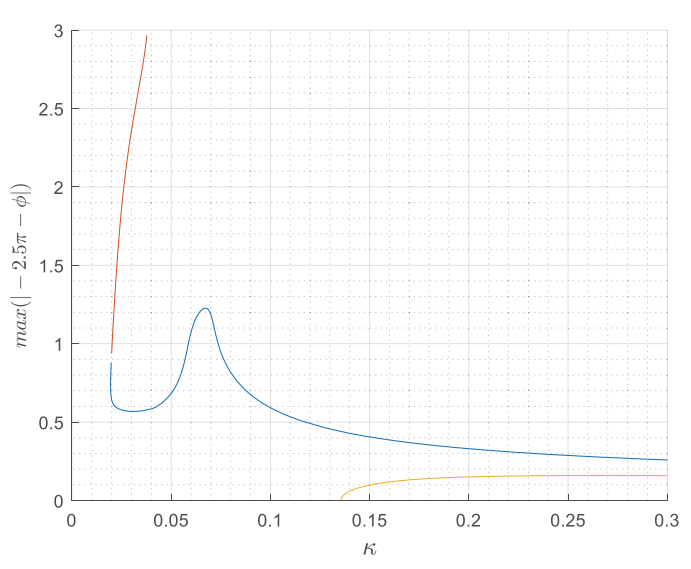

(b) Phase deviation

Figure 17. Deviations of observed dynamics from the required resonant operation mode

We see that with an increase in the quality factor, the lower $\kappa$ boundary for the region of possible multifrequency modes shifts to the left: in the case under consideration, $\kappa_{\min }=2 \%$. Unlike the case $Q=10$, here the Andronov-Hopf bifurcation is supercritical - the branching of the stable limit cycle is directed to the region of larger $\kappa$.

\subsubsection{Q-factor influence}

Of key practical interest is the continuation of the above bifurcation of the limit cycle in other parameters (first of all, in the resonator Q-factor), what would characterize the boundary of the region of existence of parasitic oscillation modes. The MATCONT software package allows us to solve such problems - to perform continuation of bifurcations of codimension 1. The expected result here is a curve on the $(\kappa, Q)$ plane, which characterizes the above-mentioned boundary of the stable operation of the PLL-AGC loop. In the present work, this problem was not completely solved due to significant numerical difficulties. Below are the results concerning the dependence of multifrequency oscillatory modes on the parameter $Q$ at a fixed value of the nonlinearity parameter $\kappa$.

Figures 18-19 show the evolution in the subspace $\{\tilde{a}, \tilde{\phi}\}$ of the limit cycle when $Q$ changes for $\kappa=2.5 \%$.

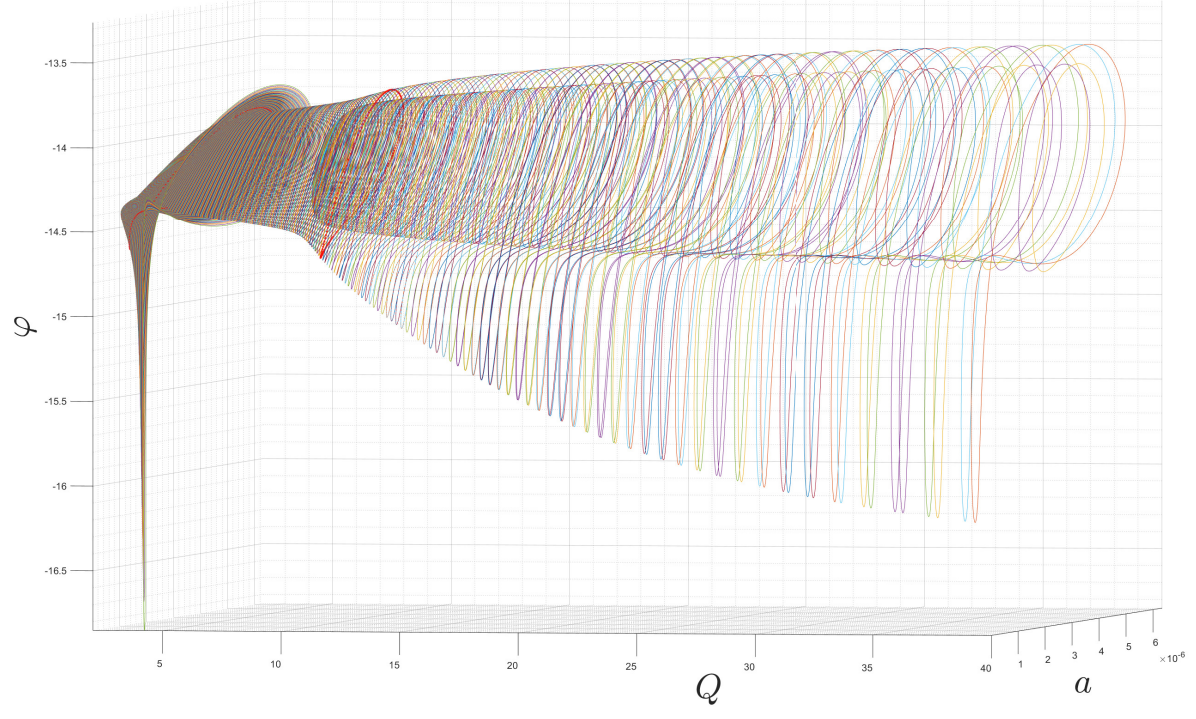

Figure 18. Evolution of the limit cycle while changing $Q$ in range $[1,40]$ 


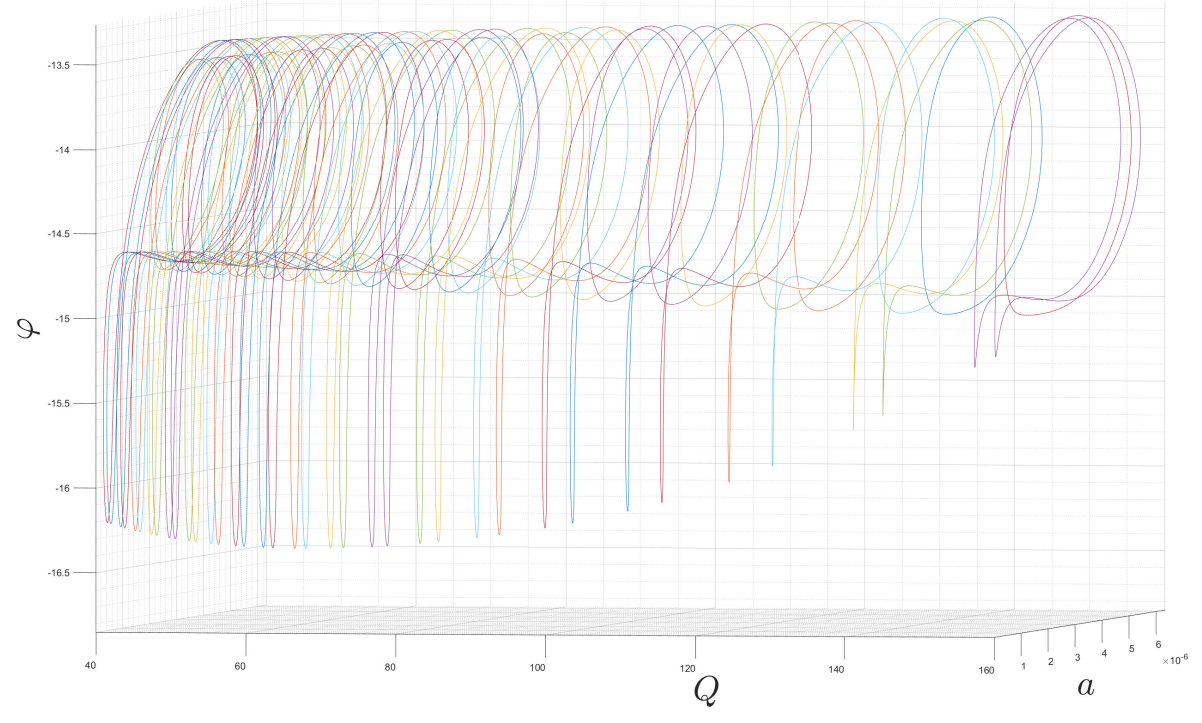

Figure 19. Evolution of the limit cycle while changing $Q$ in range $[40,160]$

Figures 20a-20b show the deviations of the amplitude and phase of the oscillations from the required resonance values.

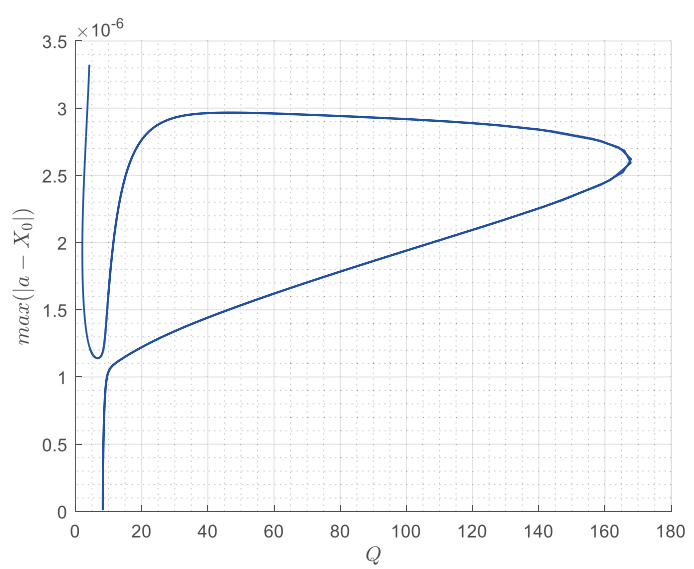

(a) Amplitude deviation

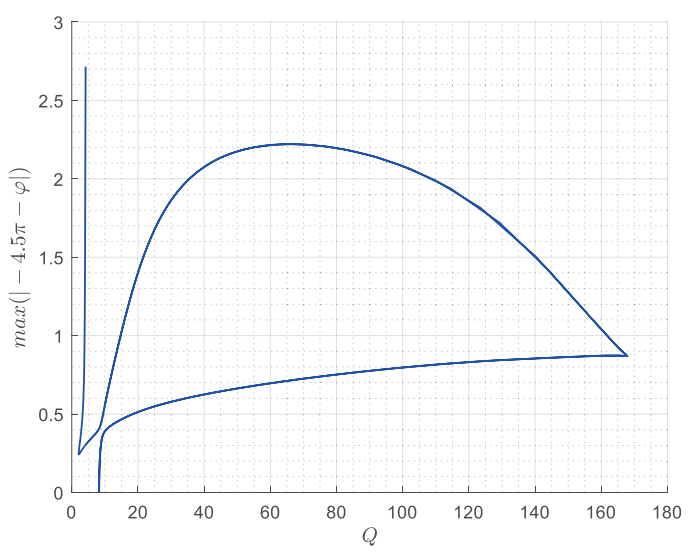

(b) Phase deviation

Figure 20. Deviations of observed dynamics from the required resonant operation mode

As can be seen from Figures 20a-20b, both the lower and the upper $Q$ boundaries of the existence of parasitic oscillatory modes are observed. It should be noted that this, generally speaking, does not imply the stability of the control loop operation outside the found zones. This is due to the fact that the numerical continuation of the limit cycle is performed only up to certain values of the active parameter; the structure of the phase space of the system under consideration is rather complex in order to expect further exits of the branches of periodic and quasi-periodic regimes beyond the found boundaries of the instability zone. For example, a direct numerical calculation of the resonator dynamics for the case corresponding to the above figures and for $Q=200$ showed an increase in the amplitude of oscillations to levels significantly exceeding the required amplitude $X_{0}$ (Figures 21-22b). 

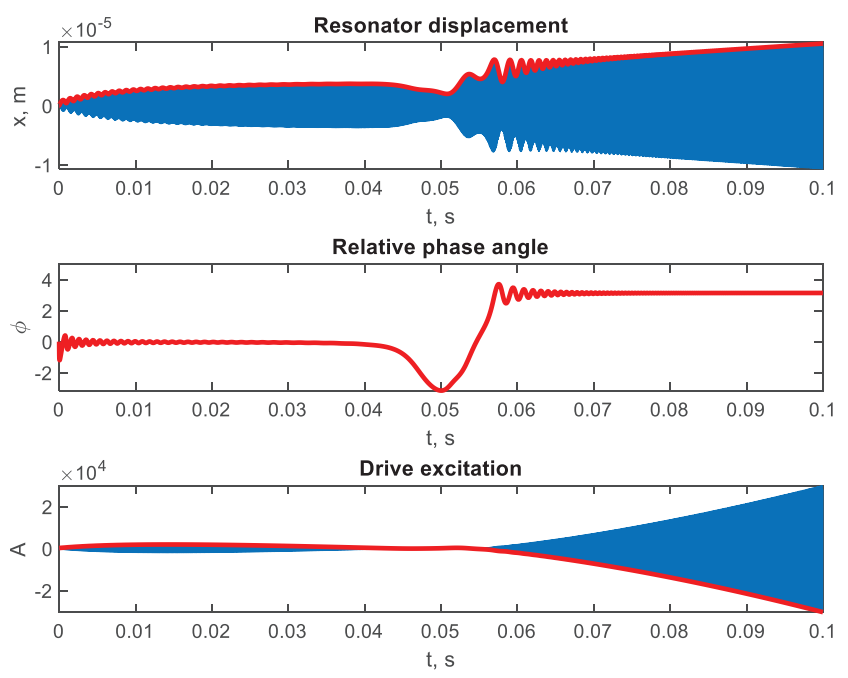

Figure 21. Dynamics of the resonator
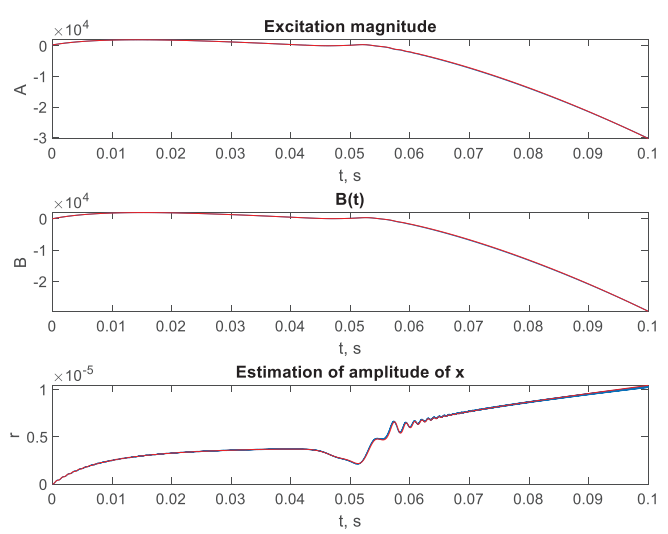

(a) AGC loop
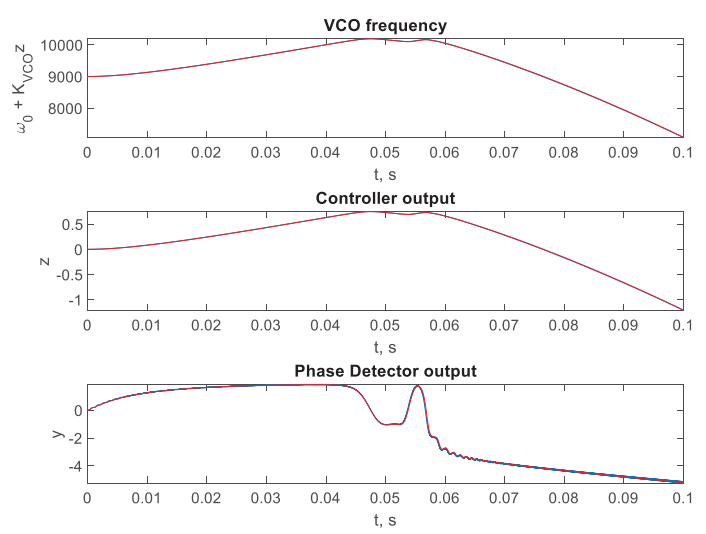

(b) PLL loop

Figure 22. Control circuit dynamics. $\kappa=2.5 \%$

The results presented above confirm the complexity of the dynamics of the system at high values of the quality factor and the instability of the adopted settings of the PLL-AGC loop.

It is noteworthy that the dynamics of the system has a similar character at $Q=140$ (at the value for which, according to the Figures 20a-20b, two-frequency oscillations around the required resonance mode were found). This indicates the multiplicity of possible dynamic modes for the selected parameter values (among which the $\kappa$ parameter is of great importance). Note that by choosing the initial conditions close to the resonant mode, it is possible to obtain the motions shown in the above figures.

\subsubsection{Analysis of the influence of the PLL and AGC loops settings}

In the course of the research it was found that the values of the poles frequencies of the low-pass filters $\lambda^{A G C}, \lambda^{P L L}$ have a significant effect on the operation of oscillations control system. In this regard, the dynamics of the system was considered in detail when these parameters were being changed. It is assumed in the calculation that $\lambda^{A G C}=\lambda^{P L L}=\lambda$. As noted earlier, $f_{n} / 10$ was taken as the base frequency value $\lambda$. The following Figures $23-24 \mathrm{~b}$ show the results of calculating the evolution of the limit cycle for $Q=10, \kappa=2.5 \%$ depending on $n_{\lambda}$, where $\lambda=\frac{f_{n}}{n_{\lambda}}$. 


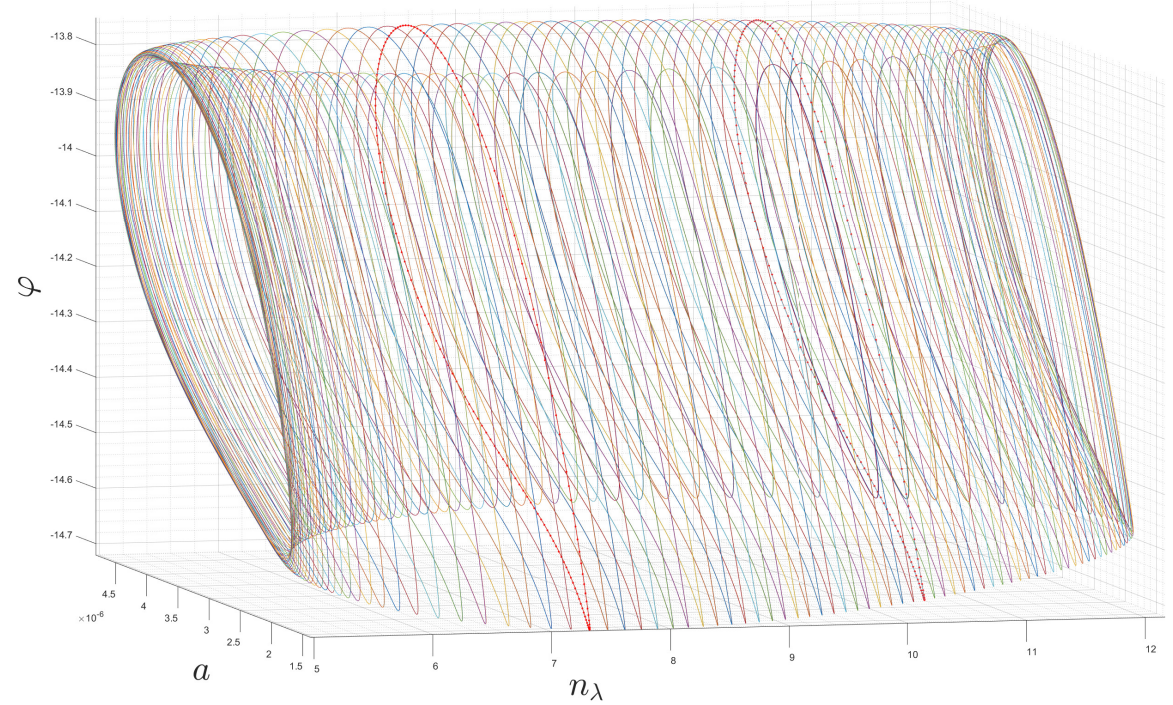

Figure 23. Evolution of the limit cycle while changing $n_{\lambda}$

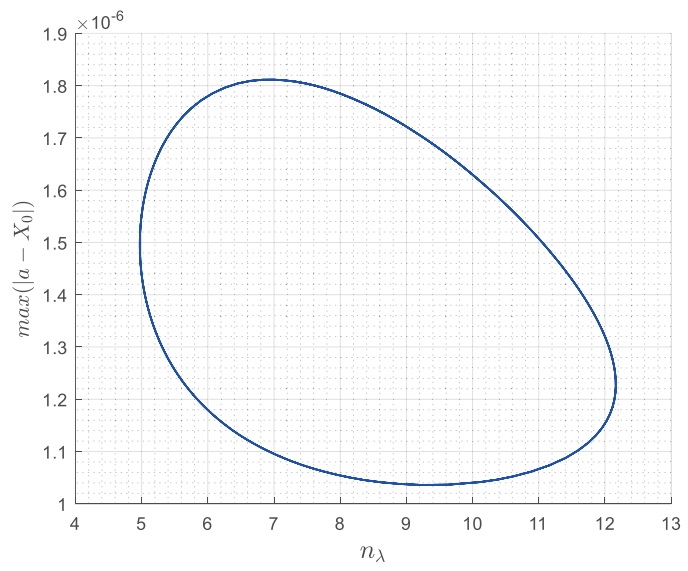

(a) Amplitude deviation

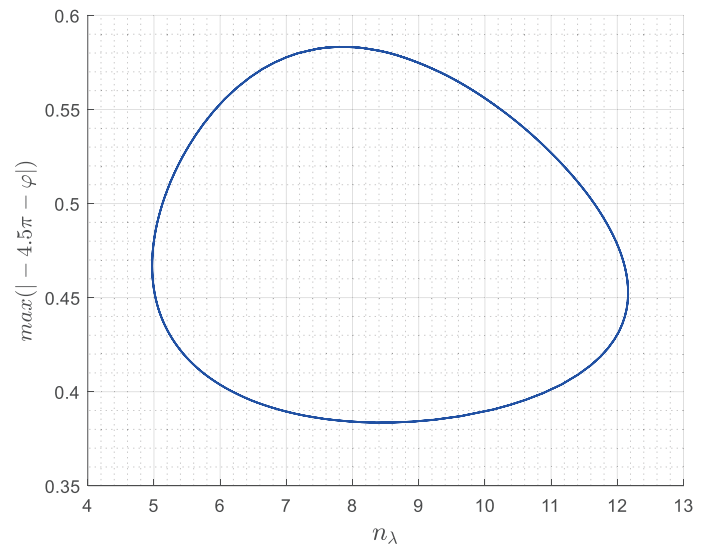

(b) Phase deviation

Figure 24. Deviations of observed dynamics from the required resonant operation mode

As can be seen from the figures, the found branch of periodic solutions (limit cycles) is closed. System dynamics when $n_{\lambda}$ is out of range $\approx[5,13]$ is subject to special consideration. Figure 25 shows the results of numerical simulation for various values $n_{\lambda}$. 

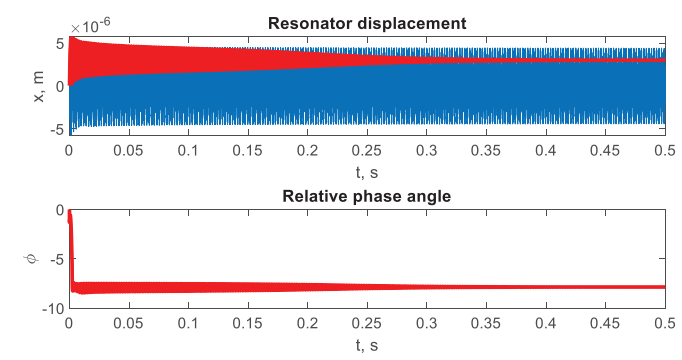
Drive excitation

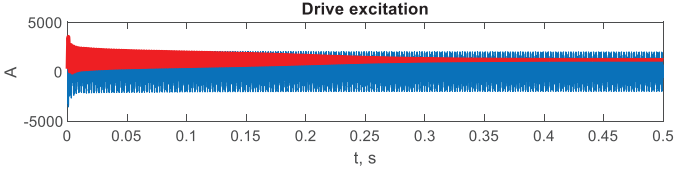

(a) $n_{\lambda}=4$
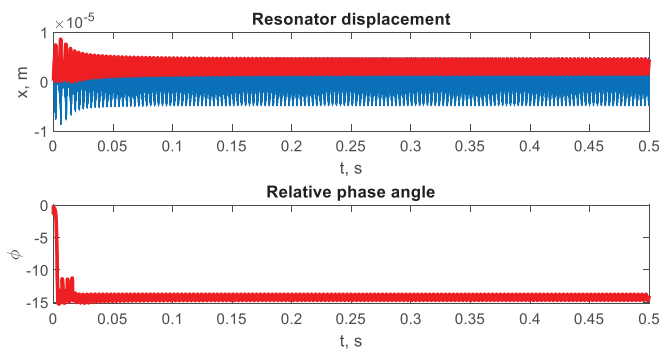

Drive excitation

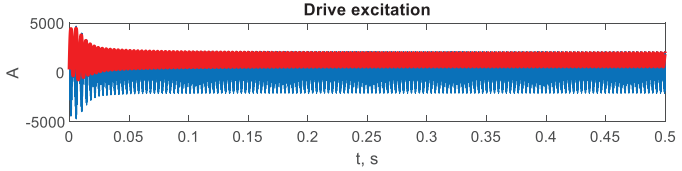

(c) $n_{\lambda}=10$

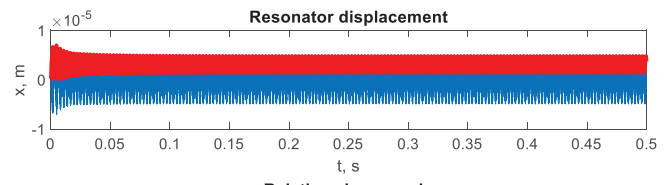

Relative phase angle

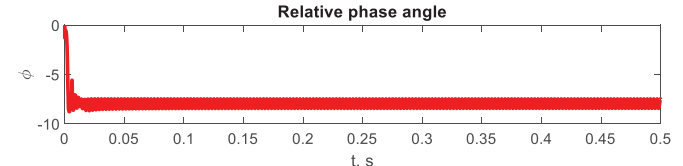

Drive excitation

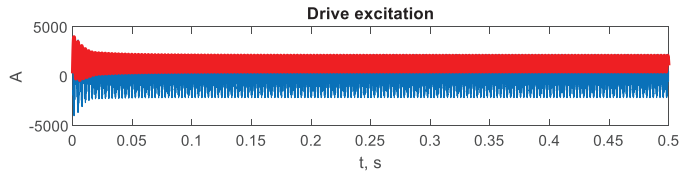

(b) $n_{\lambda}=7$

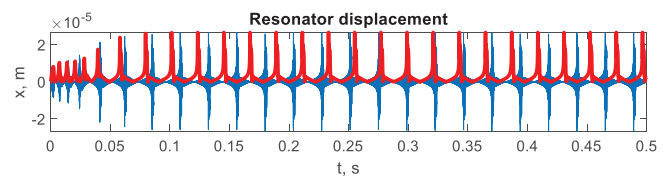

Relative phase angle

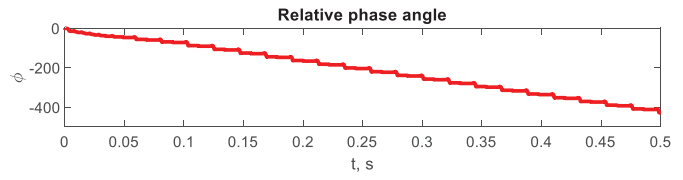

Drive excitation

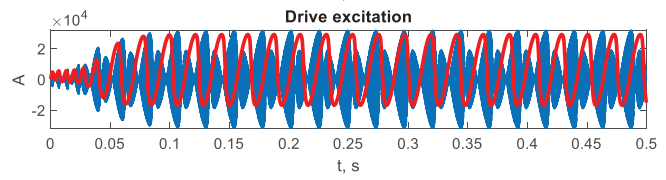

(d) $n_{\lambda}=13$

Figure 25. Dynamics of the resonator at various $n_{\lambda}$

It is noteworthy that with a decrease in the filter frequency $\lambda$ (with an increase in $n_{\lambda}$ to a value of 13), the control system does not provide stable resonator dynamics; on the contrary, the stationary mode with an amplitude close to $X_{0}$ is achieved by increasing $\lambda\left(n_{\lambda}=4\right)$. It is also important to note that an increase in the frequency of the low-pass filter leads to a gradual violation of the initial assumption about the slowness of the phase detector variable $y$ compared to the phase variable $\theta$, which in turn leads to a decrease in the accuracy of the results obtained when studying the averaged system (8).

\section{Conclusions}

This work presents the results of a qualitative study of the dynamics of a micromechanical resonator taking into account the action of a control system with PLL and AGC loops. Particular attention was directed to studying the features associated with the presence of a nonlinear component of the elastic restoring force of the resonator. It has been found that the choice of control system parameters based on the analysis of the stability of the operating resonance mode, in general case, does not provide the required stabilization of the amplitude and relative phase of oscillations. Numerical simulation revealed attractive (stable) complex multifrequency oscillation modes with variable amplitude and phase. An analytical study of the mechanisms of the appearance of these modes and their evolution when changing the key parameters of the system: the degree of mechanical nonlinearity, the Q-factor of the resonator and the frequency of the poles of the low-pass filter of the PLL and AGC circuits is carried out. For the considered computational cases, the real regions of stable operation of the control system are determined (which, as was found, do not coincide with 
the regions of stability of the operating resonance mode, due to the presence of hidden attractors in the phase space of the system). A technique has been developed for determining such areas of stable operation for specific parameters of the considered real systems. Significant difficulties associated with the qualitative analysis of the nonlinear dynamics of high-Q controlled resonators have been revealed. However, for this case, the models presented above give accurate numerical results that are of immediate practical importance.

Author Contributions: Conceptualization, A. Lukin, D. Indeitsev and Y. Belyaev; methodology, A. Lukin and I. Popov; software, A. Lukin and I. Popov; validation, Y. Belyaev; formal analysis, A. Lukin, D. Indeitsev and I. Popov; investigation, A. Lukin and I. Popov; resources, Y. Belyaev; data curation, I. Popov; writing-original draft preparation, A. Lukin; writing-review and editing, D. Indeitsev and I. Popov; visualization, A. Lukin; supervision, D. Indeitsev.

Funding: This research received no external funding

Conflicts of Interest: The authors declare no conflict of interest.

\section{Abbreviations}

The following abbreviations are used in this manuscript:

MEMS Microelectromechanical systems

AGC Automatic gain control

PLL Phase-locked loop

VCO Voltage-controlled oscillator

LPF Low-pass filter

\section{References}

1. Alper, S.; Sahin, K.; Akin, T. An Analysis to Improve Stability of Drive-Mode Oscillations in Capacitive Vibratory MEMS Gyroscopes. 2009, pp. 817 - 820. doi:10.1109/MEMSYS.2009.4805508.

2. Dong, L.; Avanesian, D. Drive-Mode Control for Vibrational MEMS Gyroscopes. Industrial Electronics, IEEE Transactions on 2009, 56, 956 - 963. doi:10.1109/TIE.2008.2010088.

3. Zhu, H.; Jin, Z.; Hu, S.; Ma, W.; Liu, Y. Drive mode control for MEMS gyroscopes with electrostatically tunable structure. 2013, pp. 273-276. doi:10.1109/3M-NANO.2013.6737430.

4. Tamara, N.; E.s, B.; Baranov, P. Amplitude control system of drive-mode oscillations of MEMS gyroscopes. IOP Conference Series: Materials Science and Engineering 2019, 516, 012009. doi:10.1088/1757-899X/516/1/012009.

5. Demir A.; Hanay M. S. Phase-Locked Loop based Resonant Sensors: A Rigorous Theory and General Analysis Framework for Deciphering Fundamental Sensitivity Limitations due to Noise. arXiv: Applied Physics 2019.

6. Wu, H.; Yang, H.g.; Yin, T.; Zhang, H. Stability analysis of MEMS gyroscope drive loop based on CPPLL. 2011, pp. 45-48. doi:10.1109/PrimeAsia.2011.6075067.

7. Lyukshonkov R.G. Thermal compensation in micromechanical gyroscopes with a circuit for stabilizing the amplitude of primary oscillations. St. Petersburg. ITMO University 2016. Dissertation for the degree of candidate of technical sciences [in russian].

8. M'Closkey, R.; Vakakis, A. Analysis of a microsensor automatic gain control loop. 1999, Vol. 5, pp. 3307 3311 vol.5. doi:10.1109/ACC.1999.782377.

9. Sun, X.; Horowitz, R.; Komvopoulos, K. Stability and Resolution Analysis of a Phase-Locked Loop Natural Frequency Tracking System for MEMS Fatigue Testing. Journal of Dynamic Systems Measurement and Control-transactions of The Asme 2002, 124. doi:10.1115/1.1514658.

10. Park, S.; Tan, C.W.; Kim, H.; Hong, S. Oscillation Control Algorithms for Resonant Sensors with Applications to Vibratory Gyroscopes. Sensors (Basel, Switzerland) 2009, 9, 5952-67. doi:10.3390/s90805952.

11. Kuznetsov, N.; Leonov, G.; Yuldashev, M.; Yuldashev, R. Hold-In, Pull-In, and Lock-In Ranges of PLL Circuits: Rigorous Mathematical Definitions and Limitations of Classical Theory. IEEE Transactions on Circuits and Systems I: Regular Papers 2015, 62. doi:10.1109/TCSI.2015.2476295. 
12. Ponomarenko, V. Complicated dynamic regimes in phase-controlled self-excited oscillation system. Journal of Communications Technology and Electronics 2017, 62, 1136-1147. doi:10.1134/S1064226917100114.

13. Kuznetsov, N.; Leonov, G.; Yuldashev, M.; Yuldashev, R. Hidden attractors in dynamical models of phase-locked loop circuits: Limitations of simulation in MATLAB and SPICE. Communications in Nonlinear Science and Numerical Simulation 2017, 51. doi:10.1016/j.cnsns.2017.03.010.

14. Nabholz, U.; Curcic, M.; Mehner, J.; Degenfeld-Schonburg, P. Nonlinear Dynamical System Model for Drive Mode Amplitude Instabilities in MEMS Gyroscopes. 2019, pp. 1-4. doi:10.1109/ISISS.2019.8739703.

15. Su, Y.; Xu, P.; Han, G.; Si, C.; Ning, J.; Yang, F. The Characteristics and Locking Process of Nonlinear MEMS Gyroscopes. Micromachines 2020, 11, 233. doi:10.3390/mi11020233.

16. Miller, J.; Shin, D.; Kwon, H.K.; Shaw, S.; Kenny, T. Phase Control of Self-Excited Parametric Resonators. Physical Review Applied 2019, 12. doi:10.1103/PhysRevApplied.12.044053.

17. Shahgildyan V.V.; Lyakhovkin A.A. Phase-locked loop systems; M.: Communication, 1972. [in russian].

18. Dhooge, A.; Govaerts, W.; Kuznetsov, Y. MATCONT: a Matlab package for numerical bifurcation analysis of ODEs. ACM Trans. Math. Softw. 2003, 29, 141-164. doi:10.1145/980175.980184. 